\title{
ON SOME CONSTANTS IN SIMULTANEOUS APPROXIMATION
}

\author{
K. BALÁZS \\ Budapest University of Economics \\ Budapest 5, Pf. 489, II-1828 Ilungary \\ T. KILGORE \\ Division of Mathematics \\ Auburn University, Auburn, Alabama 36849 \\ (Mailing address of authors) \\ (Received March 3, 1993 and in revised form February 18, 1994)
}

\begin{abstract}
Pointwise estimates for the error which is feasible in simultaneous approximation of a function and its derivatives by an algebraic polynomial were originally pursued from theoretical motivations, which did not immediately require the estimation of the constants in such results. Ilowever, recent numerical experimentation with traditional techniques of approximation such as Lagrange interpolation, slightly modified by additional interpolation of derivatives at \pm 1 , shows that rapid convergence of an approximating polynomial to a function and of some derivatives to the derivatives of the function is often easy to achieve. The new techniques are theoretically based upon older results about feasibility, contained in work of Trigub, Gopengauz. Telyakovskii, and others, giving new relevance to the investigation of constants in these older results. We begin this investigation here. Helpful in obtaining estimates for some of the constants is a new identity for the derivative of a trigonometric polynomial, based on a well known identity of M. Riesz. One of our results is a new proof of a theorem of Gopengauz which reduces the problem of estimating the constant there to the question of estimating the constant in a simpler theorem of Trigub used in the proof.
\end{abstract}

AMS Subject Classification: Primary 41A28, 42A05, Secondary 65

KEYWORDS AND PIIRASES. Simultaneous approximation, theorem of Gopengauz 1992 AMS CLASSIFICATION CODES. PRIMARY 41, SECONDARY 65.

\section{INTRODUCTION}

There are several results which give pointwise estimates of the error in simultaneous approximation of a function $f \in C^{q}[-1,1]$ and of its $q$ derivatives by an algebraic polynomial and its corresponding derivatives, in terms of the modulus of continuity of $f^{(q)}$, which we recall is defined by

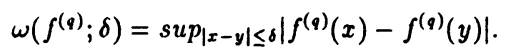

One of the first of these results was a theorem of Trigub, the relevant part of which states: 
THEOREM 1. (see Trigub [1]) Let $f \in C^{4}[-1,1]$ Then for each $n>2 q$, there exists a polynomal $P_{n}$ of degree at most $n$ such that for $k=0, \ldots, q$ and for $-1 \leq x \leq 1$

$$
\left|f^{(k)}(x)-P_{n}^{(k)}(x)\right| \leq M\left(\frac{\sqrt{1-x^{2}}}{n}+\frac{1}{n^{2}}\right)^{y-\ell} \omega\left(f^{(\varphi)} ; \frac{\sqrt{1-x^{2}}}{n}+\frac{1}{n^{2}}\right)
$$

with $M$ independent of $n$ and $f$.

A theorem of Gopengauz [2], based upon the theorem of Trigub, shows that there is the possibility of exact approximation at the endpoints \pm 1 :

THEOREM 2. Let $f \in C^{4}[-1,1]$. Then for each $n \geq 4 q+5$, there exists a polynomal $P_{n}$ of degrie at most $n$ such that for $k=0, \ldots, q$ and for $-1 \leq x \leq 1$

$$
\left|f^{(k)}(x)-P_{n}^{(k)}(x)\right| \leq K\left(\frac{\sqrt{1-x^{2}}}{n}\right)^{4-k} \omega\left(f^{(q)} ; \frac{\sqrt{1-x^{2}}}{n}\right)
$$

with $K$ independent of $n$ and $f$.

Such results as these were originally pursued for the sake of completing the theory of algebraic polynomial approximation. Theoretical interest in similar results has been widespread and sustained, leading to a literature too extensive to cite or paraphrase here. In such a theoretical context, howeves, the question of obtaining a value for the constants is not urgent. In fact a close examination of the original proofs of these results and of those in such related work as that of Telyakovskii [3] shows that the proofs are extremely uneconomical concerning constants, and the question of estimating any of the relevant constants (obviously difficult) has been little addressed in the subsequent work.

More recently, results such as the theorem of Gopengauz have been used as essential tools in showing that Lagrange interpolation (and other linear projections, too) can be modified by interpolation of some derivatives at \pm 1 with good effect: the derivatives of the function being approximated are simultancously approximated by the derivatives of the approximating polynomial, at a rate which approaches what is theoretically feasible. Evidence obtained from computer experimentation indicates that polynomial interpolation e.g. on nodes generated by Chebyshev or Jacobi polynomials, when modified by the introduction of interpolation of derivatives at \pm 1 , can give numerical results for convergence to many standard "bad" functions (the infamous Runge function and some others) and their derivatives which are very good indeed. For example, Tasche [4] and Baszenski and Tasche [5] have combined these methods with computation by a fast algorithm and have developed a method of approximation which seems comparable in its practical efficiency to cubic spline approximation and seems to give superior approximation for a comparable number of data points if the function is several times differentiable.

Thus, there is the potential of applying new methods of simultaneous approximation in numerical mathematics, but estimates of the error incurred in approximation require reasonable estimates of the constants in such basic theorems on simultaneous approximation as that of Gopengauz. Ilere, we begin to address this problem with the following, result:

THEOREM 3. For a function $f \in C^{(4)}[-1,1]$ let $P_{n}$ be a polynomial satisfyıng (1.1) for some $C$ (which may or may not depend upon $n$ or $f$, as we choose) and also satisfying

$$
f^{(k)}( \pm 1)=P_{n}^{(k)}( \pm 1) \text { for } k=0, \ldots, q \text {. }
$$


Then (1.2) follows with a constant $K \leq \max \left\{4 e^{e} C, 7 C+7\right\}$. In particular, the relation between $\kappa$ and $C$ is absolute and independent of all other quantities involved.

From our theorem, the theorem of Gopengauz will also follow, once it is shown how to construct polynomials satisfying (1.1) and (1.3). We will complete the proof of (1.2) by giving a new construction for such polynomials. Thus, (1.2) will be derived directly from Trigub's (1.1), bypassing a second conclusion of Trigub's theorem (not stated here in detail) giving a pointwise estimate for $\left|P_{n}^{(y+1)}(x)\right|$. There are two reasons for this innovation. One is that the second conclusion of Trigub, while interesting in itself, is not needed here. The second is that for both the first and the second conclusion of Trigub's theorem to hold, the value of the constant $M$ in the theorem must be large enough to accommodate both conclusions, requiring the user of Trigub's theorem to begin his work with a larger value of $M$. Another fact relevant here is that the original proof of Gopengauz and also the derivation of a pointwise estimate for $P_{n}^{(q+1)}$ in Trigub's theorem depend upon an inequality of Brudnyi giving such pointwise estimates of derivatives. To date, no estimate of the constant in Brudnyi's inequality exists, either. Our proof will permit another improvement which can have importance in applications: the minimal value of $n$ for which the estimate is valid can be lowered from $4 q+5$ to $2 q+1$.

\section{A LEMMA}

The following result will be useful in proving the Theorem:

LEMMA 1. Let $T_{n}(\theta)$ be a trigonometric polynomial of degree at most $n$. Then its derivative $T_{n}^{\prime}(\theta)$ can be written as

$$
T_{n}^{\prime}(\theta)=\frac{1}{4 m} \sum_{j=1}^{2 m} T_{n}\left(\theta+t_{j}\right)\left(\frac{\sin \frac{n t_{2}}{2}}{n \sin \frac{t_{2}}{2}}\right)^{2} \frac{(-1)^{\jmath+1}}{\left(\sin \frac{1}{2} t_{\jmath}\right)^{2}}
$$

in which $m:=2 n$ and in which

$$
t,:=\frac{2 j-1}{2 m} \pi=\frac{2 j-1}{4 n} \pi \text {. }
$$

PROOF: Setting $m:=2 n$, we invoke the formula of M. Riesz [0], which gives for an arbitrary trigonometric polynomial $\Phi_{m}$ of degree at most $m$

$$
\Phi_{m}^{\prime}(z)=\frac{1}{4 m} \sum_{j=1}^{2 m} \Phi_{m}\left(z+t_{j}\right) \frac{(-1)^{j+1}}{\left(\sin \frac{1}{2} t_{j}\right)^{2}},
$$

where $t_{j}:=\frac{(2 j-1) \pi}{2 m}=\frac{(2 j-1) \pi}{4 n}$. Replacement in this identity of $\Phi_{m}(z)$ by $\frac{1}{m} \sin m z$ gives for $z=0$ the useful fact that

$$
1=\frac{1}{4 m^{2}} \sum_{j=1}^{2 m} \frac{1}{\left(\sin \frac{1}{2} t\right)^{2}} .
$$

Having noticed this, we now assume that $\psi_{n}(z)$ is an arbitrary trigonometric polynomial of degree at most $n$ and choose

$$
\Phi_{m}(z)=\psi_{n}(z)\left(\frac{\sin \frac{n z}{2}}{i \operatorname{sir} \frac{z}{2}}\right)^{2}
$$


For $z=0$ we thus obtain

$$
\psi_{n}^{\prime}(0)=\frac{1}{4 m} \sum_{j=1}^{2 m} \psi_{n}\left(t_{\jmath}\right)\left(\frac{\sin \frac{n t_{2}}{2}}{n \sin \frac{t_{2}}{2}}\right)^{2} \frac{(-1)^{\jmath+1}}{\left(\sin \frac{1}{2} t_{\jmath}\right)^{2}} .
$$

Finally, since $\psi_{n}$ was arbitrary, we may set $\psi_{n}(z):=T_{n}(z+\theta)$, obtaining (2.1), and the proof of the Lemma is complete.

\section{PROOFS}

We begin with the proof of Theorem 3, and then we will show how this result can be used in the proof of Theorem 2.

PROOF: The proof of our Theorem (Theorem 3) divides itself naturally into two cases: that $k=q$ (including the possibility that $k=q=0$ ) is one of the cases, and that $k<q$ is the other.

Case $1(k=q)$ : We prove that

$$
\left|f^{(q)}( \pm 1)-P_{n}^{(q)}( \pm 1)\right|=0 \text { and }\left|f^{(q)}(x)-P_{n}^{(q)}(x)\right| \leq C \omega\left(f^{(q)} ; \frac{\sqrt{1-x^{2}}}{n}+\frac{1}{n^{2}}\right)
$$

implies

$$
\left|f^{(q)}(x)-P_{n}^{(q)}(x)\right| \leq C^{\prime} \omega\left(f^{(q)} ; \frac{\sqrt{1-x^{2}}}{n}\right),
$$

in which $C^{\prime}$ depends only upon $C$. The special case that $q=0$ is also covered here.

Clearly, (3.1) holds with any $C^{\prime} \geq 6 C$ if $\sqrt{1-x^{2}} \geq \frac{1}{5 n}$ or if $x= \pm 1$. Therefore we will assume that $0<\sqrt{1-x^{2}}<\frac{1}{5 n}$, and there is no loss of generality in assuming further that $x$ lies near 1 . We then may make the estimates

$$
\left|f^{(q)}(x)-P_{n}^{(q)}(x)\right| \leq\left|f^{(q)}(x)-f^{(q)}(1)\right|+\left|P_{n}^{(q)}(x)-P_{n}^{(q)}(1)\right|,
$$

and

$$
\left|f^{(q)}(x)-f^{(q)}(1)\right| \leq \omega\left(f^{(q)} ; 1-x\right) \leq \omega\left(f^{(q)} ; \frac{\sqrt{1-x^{2}}}{n}\right) .
$$

A more precise pointwise estimate for $\left|P_{n}^{(q)}(x)-P_{n}^{(q)}(1)\right|$ is needed which is zero when $x=1$. To obtain such an estimate, we notice first that for arbitrary $t \in[-1,1]$

$$
\left|P_{n}^{(q)}(t)-P_{n}^{(q)}(1)\right| \leq\left|P_{n}^{(q)}(t)-f^{(q)}(t)\right|+\left|f^{(q)}(t)-f^{(q)}(1)\right|
$$

whence

$$
\left|P_{n}^{(q)}(t)-P_{n}^{(q)}(1)\right| \leq C \omega\left(f^{(q)} ; \frac{\sqrt{1-t^{2}}}{n}+\frac{1}{n^{2}}\right)+\omega\left(f^{(q)} ; 1-t\right) .
$$

We now define $\theta_{x}:=\arccos x$ and $t \leftrightarrow \cos \theta$ for $0<\theta<\theta_{x}$. Then we have $0<\sin \theta<\sin \theta_{x} \leq \frac{1}{5 n}$, and furthermore for $n \geq 1$ we have $\frac{\theta}{\sin \theta} \leq \frac{\pi}{3}$. Now we define $T_{n}(\theta):=P_{n}^{(q)}(\cos \theta)-P_{n}^{(q)}(1)$, and we note that

$$
\left|P_{n}^{(q)}(x)-P_{n}^{(q)}(1)\right| \leq \int_{0}^{\theta_{x}}\left|T_{n}^{\prime}(\theta)\right| d \theta .
$$


Using the inequality (3.4) in the identity (2.1) and noting (2.3), we can establish a pointwise estimate for $\left|T_{n}^{\prime}(\theta)\right|$ on the interval $\left[0, \theta_{x}\right]$.

$$
\begin{aligned}
\left|T_{n}^{\prime}(\theta)\right| \leq & \frac{1}{4 m} \sum_{j=1}^{2 m}\left(\frac{\left|P_{n}^{(q)}(\cos (\theta+t,))-P_{n}^{(\varphi)}(1)\right|}{\left(\sin \frac{t_{2}}{2}\right)^{2}}\right) \frac{\left(\sin \frac{n t_{2}}{2}\right)^{2}}{\left(n \sin \frac{1}{2} t,\right)^{2}} \\
& \leq 2 n \max _{j}\left(\frac{\left|P_{n}^{(q)}(\cos (\theta+t,))-P_{n}^{(q)}(1)\right|}{\left(n^{2} \sin \frac{t_{2}}{2}\right)^{2}}\right)\left(\sin \frac{n t_{1}}{2}\right)^{2} \\
& \leq 2 n \max _{j} C \omega\left(f^{(\varphi)} ; \frac{\sin (\theta+t,)}{n}+\frac{1}{n^{2}}\right) \cdot \frac{\left(\sin \frac{n t_{2}}{2}\right)^{2}}{n^{2} \sin ^{2} \frac{t_{2}}{2}} \\
& +2 n \max \omega\left(f^{(q)} ; 2 \sin ^{2} \frac{\theta+t,}{2}\right) \cdot \frac{\left(\sin \frac{n t_{2}}{2}\right)^{2}}{n^{2} \sin ^{2} \frac{t_{2}}{2}} \\
& \leq A(\theta)+B(\theta) .
\end{aligned}
$$

Now we use the fact that $\omega\left(f^{(y)} ; \lambda \delta\right) \leq(1+\lambda) \omega\left(f^{(\varphi)} ; \delta\right)$ to estimate $A(\theta)$ and then $B(\theta)$. We obtain

$$
\begin{aligned}
A(\theta) \leq & 2 n C \max _{\jmath}\left(1+\frac{|\sin (\theta+t,)|}{\sin \theta_{x}}+\frac{1}{n \sin \theta_{x}}\right) \omega\left(f^{(\varphi)} ; \frac{\sin \theta_{x}}{n}\right) \cdot \frac{\left(\sin \frac{n t_{2}}{2}\right)^{2}}{n^{2} \sin ^{2} \frac{t_{2}}{2}} \\
& \leq 2 n C \max _{,}\left(1+\frac{|\sin \theta \cos t,|+|\cos \theta \sin t,|}{\sin \theta_{x}}+\frac{1}{n \sin \theta_{x}}\right) \omega\left(f^{(y)} ; \frac{\sin \theta_{x}}{n}\right) \cdot \frac{\left(\sin \frac{n t_{2}}{2}\right)^{2}}{n^{2} \sin ^{2} \frac{t_{2}}{2}} .
\end{aligned}
$$

Therefore

$$
\begin{gathered}
\int_{0}^{\theta_{x}} A(\theta) d \theta \leq 2 n C \max _{J}\left(\theta_{x}+\frac{\left(1-\cos \theta_{x}\right)\left|\cos t_{j}\right|}{\sin \theta_{x}}+\left|2 \sin \frac{t_{\jmath}}{2} \cos \frac{t_{J}}{2}\right|+\frac{\theta_{x}}{n \sin \theta_{x}}\right) \\
\cdot \omega\left(f^{(q)} ; \frac{\sin \theta_{x}}{n}\right) \cdot \frac{\left(\sin \frac{n t_{y}}{2}\right)^{2}}{n^{2} \sin ^{2} \frac{t_{1}}{2}},
\end{gathered}
$$

and we easily obtain

$$
\int_{0}^{\theta_{x}} A(\theta) d \theta \leq 2 n C\left(\frac{\pi}{15 n}+\frac{1}{5 n}+\frac{2}{n}+\frac{\pi}{3 n}\right) \omega\left(f^{(q)} ; \frac{\sin \theta_{x}}{n}\right)<7 C \omega\left(f^{(4)} ; \frac{\sin \theta_{x}}{n}\right) .
$$

We will now similarly estimate $B(\theta)$ and then its integral. It is helpful to notice that always if $0 \leq \theta \leq \theta_{x}$, then

$$
\cos \frac{\theta}{2}>\left|\cos \frac{t_{j}}{2}\right| \text { for all } j,
$$

which we will use in estimating one of the terms.

$$
\begin{aligned}
B(\theta) \leq & 2 n \max _{j}\left(1+\frac{2 n \sin ^{2} \frac{\theta+t_{2}}{2}}{\sin \theta_{x}}\right) \omega\left(f^{(q)} ; \frac{\sin \theta_{x}}{n}\right) \cdot \frac{\left(\sin \frac{n t_{2}}{2}\right)^{2}}{n^{2} \sin ^{2} \frac{t_{2}}{2}} \\
\leq & 2 n \max _{j}\left[1+2 n\left(\frac{\frac{1}{4} \sin ^{2} \theta+\sin \theta\left|\sin \frac{t_{2}}{2} \cos \frac{t_{2}}{2}\right|+\cos ^{2} \frac{\theta}{2} \sin ^{2} \frac{t_{2}}{2}}{\sin \theta_{x}}\right)\right] \\
& \cdot \omega\left(f^{(q)} ; \frac{\sin \theta_{x}}{n}\right) \cdot \frac{\left(\sin \frac{n t_{2}}{2}\right)^{2}}{n^{2} \sin ^{2} \frac{t_{2}}{2}} .
\end{aligned}
$$

Now we may estimate

$$
\begin{aligned}
& \int_{0}^{\theta_{x}} B(\theta) d \theta \leq 2 n \max _{j} {\left[\theta_{x}+2 n\left(\frac{1}{4}\left(1-\cos \theta_{x}\right)+\frac{\left(1-\cos \theta_{x}\right)\left|\sin \frac{t_{2}}{2}\right|+\frac{1}{2}\left(\theta_{x}+\sin \theta_{x}\right) \sin ^{2} \frac{t_{2}}{2}}{\sin \theta_{x}}\right)\right] } \\
& \cdot \omega\left(f^{(4)} ; \frac{\sin \theta_{x}}{n}\right) \cdot\left(\frac{\left(\sin \frac{n t_{2}}{2}\right)^{2}}{n^{2} \sin ^{2} \frac{t_{2}}{2}}\right) .
\end{aligned}
$$

From this we obtain

$$
\int_{0}^{\theta_{x}} B(\theta) d \theta \leq 2 n\left[\frac{\pi}{15 n}+2 n\left(\frac{1}{100 n^{2}}+\frac{1}{5 n^{2}}+\frac{\pi}{6 n^{2}}+\frac{1}{2 n^{2}}\right)\right] \omega\left(f^{(q)} ; \frac{\sin \theta_{x}}{n}\right) \leq 6 \omega\left(f^{(q)} ; \frac{\sin \theta_{x}}{n}\right) .
$$


The estimate for $\left|P_{n}^{(q)}(x)-P_{n}^{(4)}(1)\right|$ may now be obtained by combining (3.6) and (3.7):

$$
\left|P_{n}^{(4)}(x)-P_{n}^{(q)}(1)\right| \leq(7 C+6) \omega\left(f^{(q)} ; \frac{\sin \theta_{x}}{n}\right) .
$$

Now, combining the estimates (3.2) and (3.3) and (3.8), we have shown that

$$
\left|f^{(4)}(x)-P_{n}^{(q)}(x)\right| \leq(7 C+7) \omega\left(f^{(q)} ; \frac{\sqrt{1-x^{2}}}{n}\right)
$$

provided that $0<\sqrt{1-x^{2}} \leq \frac{1}{5 n}$, and clearly this estimate is dominant on the rest of the interval $[-1,1]$ as well. We have established (3.1) with a constant $C^{\prime}<7 C+7$. This completes the argument for Case 1 .

Case 2: It remains to show for $q \geq 1$ that if the polynomials $P_{n}$ satisfy (1.1) and (1.3) with constant $C$ then for $k=0, \ldots, q-1$ we can obtain

$$
\left|f^{(k)}(x)-P_{n}^{(k)}(x)\right| \leq C^{\prime \prime}\left(\frac{\sqrt{1-x^{2}}}{n}\right)^{\varphi-k} \omega\left(f^{(q)} ; \frac{\sqrt{1-x^{2}}}{n}\right),
$$

in which $C^{\prime \prime} \leq 4 \min \left\{2^{4-k}, e\right\} C$.

Clearly, (3.9) holds with constant $C^{\prime \prime}=2 q-k C$ if $\sqrt{1-x^{2}} \geq \frac{1}{n}$, and we need to consider those values of $x$ such that $0<\sqrt{1-x^{2}}<\frac{1}{n}$. Without any loss of generality we can assume that $x$ lies near 1 . We then have for $k \leq q$

$$
\frac{\left|f^{(k)}(x)-P_{n}^{(k)}(x)\right|}{\left(\sqrt{1-x^{2}}\right)^{q-k}}=\frac{\left|f^{(k)}(x)-P_{n}^{(k)}(x)\right|}{\left(1-x^{2}\right)^{q-k}}\left(\sqrt{1-x^{2}}\right)^{q-k} \leq \frac{C}{(q-k) !}\left|f^{(q)}(z)-P_{n}^{(q)}(z)\right| \cdot\left(\frac{1}{n}\right)^{q-k}
$$

for some intermediate point $z$ lying between $x$ and 1 . We may further estimate

$$
\left|f^{(q)}(z)-P_{n}^{(q)}(z)\right| \cdot\left(\frac{1}{n}\right)^{q-k} \leq C\left(\frac{1}{n}\right)^{q-k} \omega\left(f^{(q)} ; \frac{\sqrt{1-x^{2}}}{n}+\frac{1}{n^{2}}\right),
$$

in which we have also used the fact that $\sqrt{1-z^{2}} \leq \sqrt{1-x^{2}}$. Therefore

$$
\left|f^{(k)}(x)-P_{n}^{(k)}(x)\right| \leq 2^{q-k} C\left(\frac{\sqrt{1-x^{2}}}{n}\right)^{q-k} \omega\left(f^{(q)} ; \frac{\sqrt{1-x^{2}}}{n}+\frac{1}{n^{2}}\right) .
$$

To show that $2^{q-k} C$ can be replaced (independently of $q$ ) by $e C$, we repeat the argument, noting that if $0<\sqrt{1-x^{2}}<\frac{\left((q-k)^{\prime}\right)^{\frac{1}{q-k}}}{n}$, then

$$
\frac{\left|f^{(k)}(x)-P_{n}^{(k)}(x)\right|}{\left(\sqrt{1-x^{2}}\right)^{q-k}} \leq C\left|f^{(q)}(z)-P_{n}^{(q)}(z)\right| \cdot\left(\frac{1}{n}\right)^{q-k} .
$$

On the other hand, if $\sqrt{1-x^{2}} \geq \frac{\left((q-k)^{1}\right)^{\frac{1}{9-k}}}{n}$, we obtain

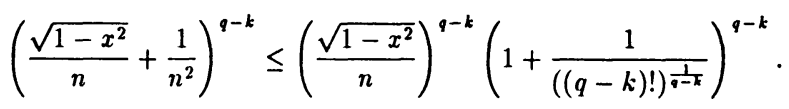

From Stirling's formula, one may note that

$$
(q-k) ! \geq e^{k-q}(q-k)^{q-k}
$$

whence

$$
\frac{1}{((q-k) !)^{\frac{1}{q-k}}} \leq \frac{e}{q-k}
$$

Combining the previous estimates, we have shown

$$
\left|f^{(k)}(x)-P_{n}^{(k)}(x)\right| \leq \min \left\{2^{q-k}, e^{e}\right\} C\left(\frac{\sqrt{1-x^{2}}}{n}\right)^{q-k} \omega\left(f^{(q)} ; \frac{\sqrt{1-x^{2}}}{n}+\frac{1}{n^{2}}\right) .
$$


Finally, it is clear that (3.11) implies (3.9) if $\sqrt{1-x^{2}} \geq \frac{1}{n}$. On the other hand, if $\sqrt{1-x^{2}} \leq \frac{1}{n}$, then we may use the well known fact that

$$
\delta^{-1} \omega\left(f^{(\varphi)} ; \delta\right) \leq 2 \epsilon^{-1} \omega\left(f^{(\varphi)} ; \epsilon\right) \text { when } 0<\delta<\epsilon
$$

and the mean value theorem of Lagrange to establish

$$
\frac{\left|f^{(k)}(x)-P_{n}^{(k)}(x)\right|}{\left(1-x^{2}\right)^{4-k}} \leq 2 \min \left\{2^{4-k}, e^{e}\right\} C\left(\frac{\sqrt{1-x^{2}}}{n}+\frac{1}{n^{2}}\right) \frac{\omega\left(f^{(4)} ; \frac{\sqrt{1-x^{2}}}{n}\right)}{\frac{\sqrt{1-x^{2}}}{n}},
$$

from which

$$
\begin{aligned}
\left|f^{(k)}(x)-P_{n}^{(k)}(x)\right| \leq & 2 \min \left\{2^{y-k}, e^{e}\right\} C\left(\sqrt{1-x^{2}}\right)^{2 q-2 k-1} \cdot n \cdot\left(\frac{\sqrt{1-x^{2}}}{n}+\frac{1}{n^{2}}\right) \omega\left(f^{(q)} ; \frac{\sqrt{1-x^{2}}}{n}\right) \\
& \leq 2 \min \left\{2^{q-k}, e^{e}\right\} C\left(\sqrt{1-x^{2}}\right)^{q-k}\left(\frac{1}{n}\right)^{q-k-1} \cdot n \cdot \frac{2}{n^{2}} \omega\left(f^{(q)} ; \frac{\sqrt{1-x^{2}}}{n}\right),
\end{aligned}
$$

and we have established (3.9).

This argument completes the proof of Case 2.

The constant $K$ in Theorem 3 can be defined as $\max \left\{C^{\prime}, C^{\prime \prime}\right\}$, and Theorem 3 is proven.

Next, we show how our Theorem 3 can be used to establish the Theorem of (iopengauz (Theorem 2):

PROOF: In view of our result (Theorem 3), it is only necessary to construct polynomials $P_{n}$ which satisfy (1.1) and also satisfy (1.3). Our method will be, beginning with polynomials $Q_{n}$ which satisfy (1.1), to construct polynomials $Q_{n, 0}, \ldots, Q_{n, q}$ in recursive fashion, and for each $j=0, \ldots, q$ the polynomial $Q_{n, j}$ will be seen still to satisfy (1.1) (with a new constant $M$, depending on $j$ ) and to satisfy

$$
\left(f^{(k)}-Q_{n, j}^{(k)}\right)( \pm 1)=0 \text { for } k=0, \ldots, j
$$

Finally, the polynomials $P_{n}=Q_{n, q}$ are seen to satisfy (1.1) with a new constant $M_{q}$ replacing the original constant $M$ and at the same time to satisfy (1.3).

We define $Q_{n, 0}$ by

$$
Q_{n, 0}(x)=Q_{n}(x)+\left(f(-1)-Q_{n}(-1)\right)\left(\frac{1-x}{2}\right)+\left(f(1)-Q_{n}(1)\right)\left(\frac{1+x}{2}\right) .
$$

Then both conclusions surely follow for $j=0$, and we have $M_{0} \leq 2 M$.

Before constructing $Q_{n, 1}, \ldots, Q_{n, q}$, we denote by $m$ the greatest even integer such that $m q<n$, and we let $T_{m}(x):=\cos (m \arccos x)$, the Chebyshev polynomial of degree $m$. The properties of $T_{m}$ which we need here are that $T_{m}( \pm 1)=1 ;\left\|T_{m}\right\| \leq 1 ;$ and $T_{m}^{\prime}(1)=m^{2} ; T_{m}^{\prime}(-1)=-m^{2}$. Now, assuming that for a given $j \in\{1, \ldots, q\}$ the polynomial $Q_{n, j-1}$ has already been defined, we let

$$
\begin{aligned}
Q_{n, j}(x):= & Q_{n, j-1}(x)+\left\{(-1)^{\jmath}\left(f^{(\jmath)}(-1)-Q_{n, j-1}^{(\jmath)}(-1)\right)\left(\frac{1-x}{2}\right)+\left(f^{(\jmath)}(1)-Q_{n, j-1}^{(\jmath)}(1)\right)\left(\frac{1+x}{2}\right)\right\} \\
& \cdot \frac{(-1)^{\jmath}}{j ! m^{2 j}}\left(1-T_{m}(x)\right)^{\jmath} \\
= & Q_{n,-1}(x)+R_{n, j}(x) .
\end{aligned}
$$

It is then seen easily that

$$
\left\|R_{n, \jmath}\right\| \leq M_{-1}\left(\frac{1}{n^{2}}\right)^{q-\jmath} \omega\left(f^{(q)} \frac{1}{n^{2}}\right) \cdot\left(\frac{2^{\jmath}}{j ! m^{2 \jmath}}\right)=M_{\jmath-1}\left(\frac{1}{n^{2}}\right)^{q} \omega\left(f^{(q)} ; \frac{1}{n^{2}}\right) \cdot \frac{1}{\jmath !} \cdot\left(\frac{2 n}{m}\right)^{\prime} .
$$


Therefore, we obtain for $k=0, \ldots, n$ (using the Markov inequality to estimate the derivatives of $R_{n, j}$ )

$$
\left|f^{(\ell)}(x)-Q_{n, j}^{(\ell)}(x)\right| \leq M,\left(\frac{\sqrt{1-x^{2}}}{n}+\frac{1}{n^{2}}\right)^{\varphi-\ell} \omega\left(f^{(4)} ; \frac{\sqrt{1-x^{2}}}{n}+\frac{1}{n^{2}}\right),
$$

in which

$$
M, \leq M_{,-1} \cdot\left(1+\frac{1}{\jmath !} \cdot\left(\frac{2 n}{m}\right)^{\prime}\right) .
$$

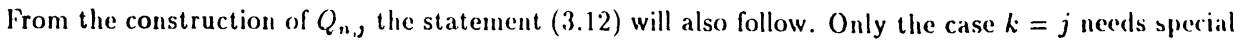
attention; we note that the derivative $R_{n, j}^{(j)}$ has exactly one term in its expansion which is not zero at \pm 1 , namely

$$
\left\{(-1)^{\jmath}\left(f^{(\jmath)}(-1)-Q_{n, \jmath-1}^{(\jmath)}(-1)\right)\left(\frac{1-x}{2}\right)+\left(f^{(\jmath)}(1)-Q_{n, J-1}^{(\jmath)}(1)\right)\left(\frac{1+x}{2}\right)\right\} \cdot \frac{(-1)^{\jmath}}{m^{2 \jmath}}\left(-T_{m}^{\prime}(x)\right)^{\jmath} .
$$

The value of this term at 1 is

$$
\left(f^{(\jmath)}(1)-Q_{n, \jmath-1}^{(\jmath)}(1)\right) \cdot \frac{\left(m^{2}\right)^{\jmath}}{m^{2 \jmath}}=f^{(\jmath)}(1)-Q_{n, \jmath-1}^{(\jmath)}(1),
$$

and its value at -1 is

$$
(-1)^{\jmath}\left(f^{(\jmath)}(-1)-Q_{n, j-1}^{(\jmath)}(-1)\right) \cdot \frac{(-1)^{\jmath}\left(m^{2}\right)^{\jmath}}{m^{2 \jmath}}=f^{(\jmath)}(-1)-Q_{n, j-1}^{(\jmath)}(-1) .
$$

Thus it follows that $f^{(\jmath)}( \pm 1)-Q_{n, j}^{(\jmath)}( \pm 1)=0$. As previously stated, we now let $P_{n}:=Q_{n, q}$. An estimate for the constant $M_{q}$ may now be obtained by noting that the following relation follows from (3.13)

$$
M_{q} \leq M \prod_{j=0}^{q}\left(1+\frac{1}{j !} \cdot\left(\frac{2 n}{m}\right)^{\prime}\right) .
$$

At this point, the proof of the theorem of Gopengauz is completed by applying the theorem of this article (Theorem 3), using the value of $C:=M_{\varphi}$. Theorem 3 then provides an estimate for the constant in the theorem of Gopengauz.

\section{REFERENCES}

1. R. TRIGUB, Approximation of functions by polynomials with integral coefficients (in Russian), Isv. Akad. Nauk. SSSR, Ser. Matem. 26 (1962), 261-280.

2. I. GOPENGAUZ, A theorem of A. F. Timan on the approximation of functions by polynomials on a finite segment, Mat. Zamelki 1 (1967), 163-172.

3. S. TELYAKOVSKII, Two theorems on the approximation of functions by algebraic polynomials, Mat. Sb. 70 (112) (1966), 252-265.

4. M. TASCHE, Fast algorithms for discrete Chebyshev-Vandermonde transforms and applications, Proceedings of the NATO Advanced Workshop on Algorithms for Approximations, Oxford, 1992.

5. G. BASZENSKI and M. TASCIE, Fast algorithms for simultaneous polynomial approximation, in "Multivariate approximation and wavelets," K. Jetter and F. Utreras, editors, World Scientific, 1992.

6. M. RIESZ, Eine trigonometrische Interpolationsformel und einige Ungleichungen für Polynome, Jahresbericht d. Deutschen Math. Ver. 23 (1914), 354-368. 


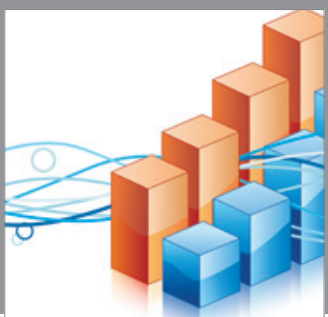

Advances in

Operations Research

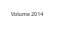

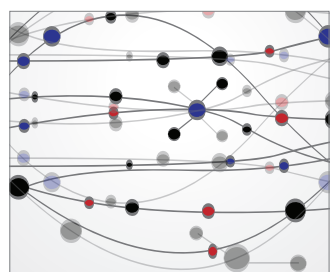

\section{The Scientific} World Journal
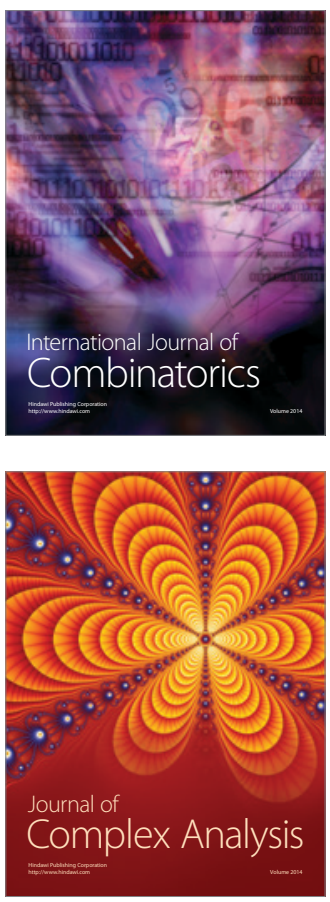

International Journal of

Mathematics and

Mathematical

Sciences
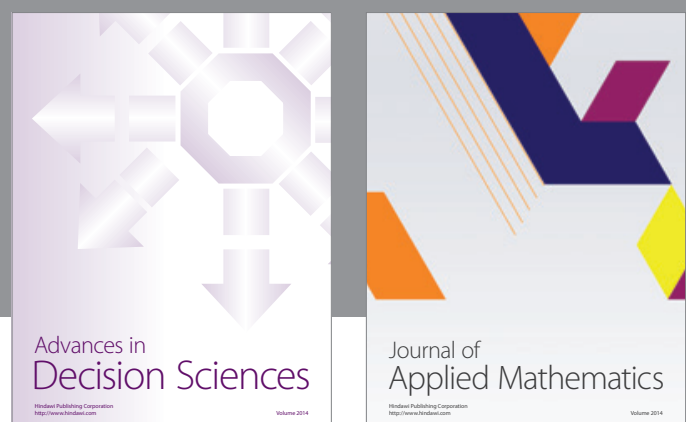

Journal of

Applied Mathematics
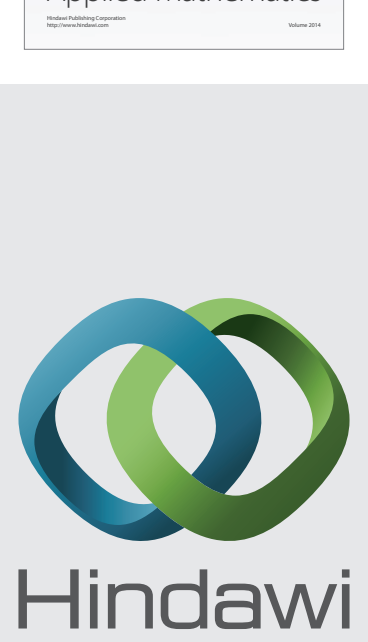

Submit your manuscripts at http://www.hindawi.com
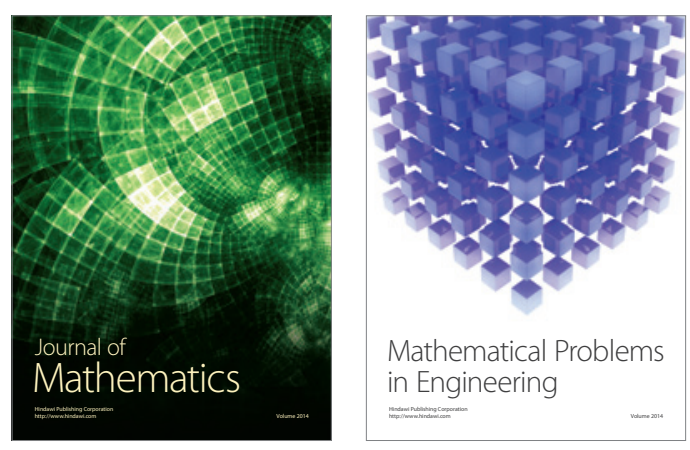

Mathematical Problems in Engineering
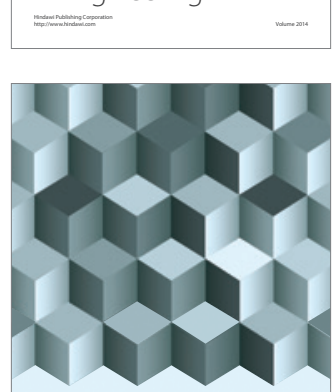

Journal of

Function Spaces
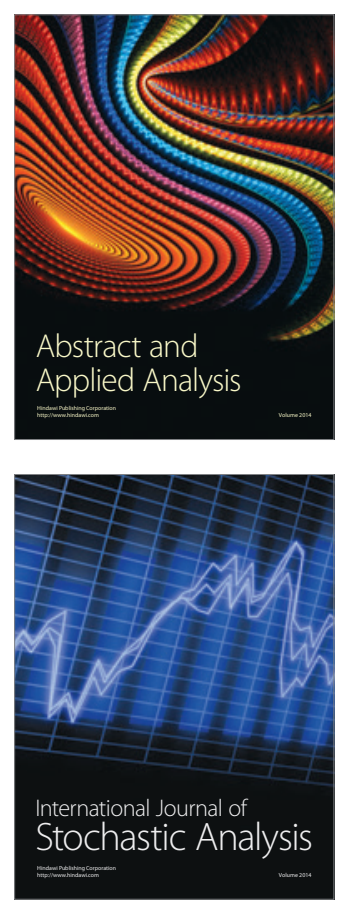

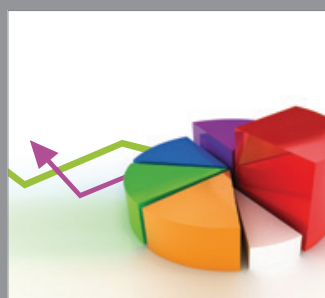

ournal of

Probability and Statistics

Promensencen
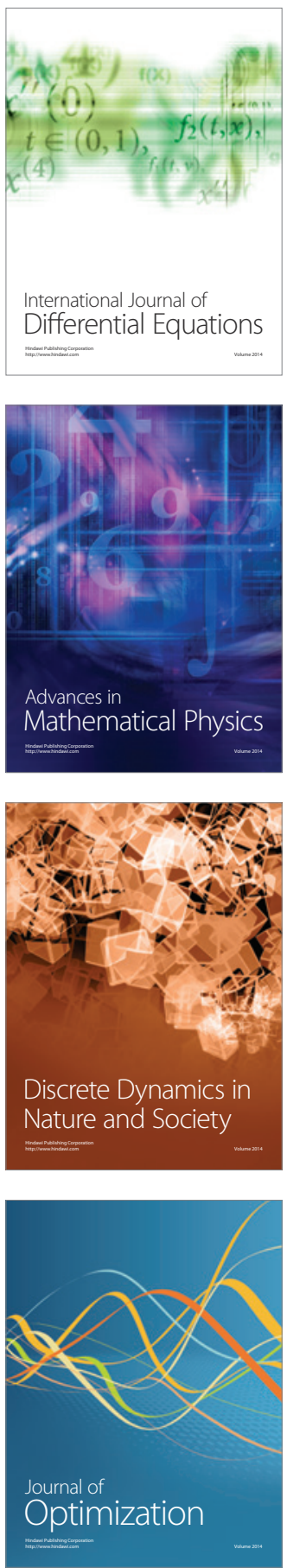